Pacific Journal of Mathematic 


\section{CANONICAL DOMAINS IN SEVERAL COMPLEX VARIABLES}

\section{SADAO KATŌ}

The main purpose of this paper is to give the function which maps a bounded domain onto the $m$-representative domain $(m \geqq 1)$ (hereafter called $m$-representative function) without utilizing the minimum problems in the case of several complex variables. One of the results obtained is that a $\mathrm{m}$ representative domain becomes also a $(m+1)$-representative domain.

Let $D$ be a bounded domain and $k_{D}(z, \bar{t}) z, t \in D$ be the Bergman kernel function. Recently M. Maschler [7] made use of the minimum problems to establish the $m$-representative function in one variable:

$$
w(z)=\frac{M_{D}^{010 \cdots 0}\left(z, t_{0}\right)}{m_{D}^{10 \cdots 0}\left(z, t_{0}\right)} \text { fixed } t_{0} \in D
$$

where $M_{D}^{010 \cdots 0}\left(z, t_{0}\right), m_{D}^{10 \cdots 0}\left(z, t_{0}\right)$ are both Maschler's minimizing functions and represented in a closed from by using $k_{D}(z, \bar{t})$ and its derivatives, respectively. Moreover this result has been generalized successfully by T. Tsuboi [13] in the case of several complex variables. In this case, however, for example the 2-representative function of a unit circle is nonregular if we choose a fixed point $t_{0}$ in $1 / 2<\left|t_{0}\right|<1$.

In this paper we consider the following $m$-representative function of other type which coincides with the ordinary Bergman representative function when $m=1(\S 4)$ :

$$
w(z)=\int_{t_{0}}^{z} N_{D}^{E} n^{0 \cdots 0}\left(z, t_{0}\right) d z,
$$

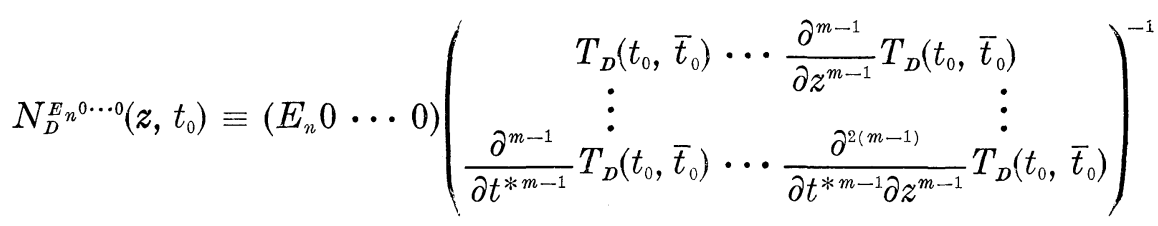

$$
\begin{aligned}
& \text { - }\left(\begin{array}{c}
T_{D}\left(z, \bar{t}_{0}\right) \\
\vdots \\
\frac{\partial^{m-1}}{\partial t^{* m-1}} T_{D}\left(z, \bar{t}_{0}\right)
\end{array}\right)
\end{aligned}
$$

where the matrix function $T_{D}(z, \bar{t})($ for definition, see $\S 1$ ) is, as is well known, relatively invariant under any pseudo-conformal mapping. We define in $\S 1$ the relative invariant $T_{2 D}(z, \bar{t})$, which plays an 
important role throughout this paper. In $\S 2$ we have a necessary and sufficient condition that a minimal domain becomes simultaneously a representative domain with the same center. Section 3 is devoted to the canonical domains of other types.

1. Preliminaries. Various properties on the several complex variables can be treated simply in many cases, considering functions as reciprocal mappings of vector spaces. In this paper we assume that each domain $D$ we deal with is a bounded domain in $n$-dimensional complex Euclidean $z \equiv\left(z_{1}, z_{2}, \cdots, z_{n}\right)^{\prime}$-space and we shall consider $w(z) \equiv$ $\left(w_{1}(z), w_{2}(z), \cdots, w_{n}(z)\right)^{\prime}$ which is called a vector function after BochnerMartin, for each point $z$ of $D$. We shall call this $w(z)$ an analytic function of $z$, if $w_{j}(z)(j=1,2, \cdots, n)$ are analytic with respect to $z$ (Ozaki and others [14]).

We start with the definitions of power of vector and differentiation with respect to vector variable. Vectors and matrices marked with the symbol ' and * denote the transposed and transposed conjugate vectors or matrices, respectively. We define the $k$-th power of $z$ as

$$
z^{k} \equiv\left(z_{1}^{k}, \cdots, z_{1}^{k_{1}} z_{2}^{k_{2}} \cdots z_{n}^{k_{n}}, \cdots, z_{n}^{k}\right)^{\prime},
$$

where $\left(k_{1}, k_{2}, \cdots, k_{n}\right)$ runs over all the nonnegative integers such that $k_{1}+k_{2}+\cdots+k_{n}=k$ and ${ }_{n} H_{k}$ monomials of degree $k$ in $z_{1}, z_{2}, \cdots$, $z_{n}$ are arranged in a certain determined way (e.g., in the lexicographical order) to form an ${ }_{n} H_{k}$-tuple column vector. Next we define the $k$-th partial differentiation of function $w(z, \bar{t})$ with respect to $z$ and $t^{*}$ as

$$
\begin{aligned}
\frac{\partial^{k}}{\partial z^{k}} w(z, \bar{t}) \equiv & \left(\frac{\partial^{k}}{\partial z_{1}^{k}}, \cdots, \frac{k !}{k_{1} ! k_{2} ! \cdots k_{n} !} \frac{\partial^{k}}{\partial z_{1}^{k_{1}} \partial z_{2}^{k_{2}} \cdots \partial z_{n}^{k_{n}}}, \cdots, \frac{\partial^{k}}{\partial z_{n}^{k}}\right) \\
& \times w(z, \bar{t})
\end{aligned}
$$

and $\partial^{k} w(z, \bar{t}) / \partial t^{* k} \equiv\left(\partial^{k} / \partial t^{k}\right)^{*} \times w(z, \bar{t})$, where $\partial^{k} / \partial z_{1}^{k}, \cdots, \partial^{k} / \partial z_{n}^{k}$ are arranged in the order corresponding to $z_{1}^{k}, \cdots, z_{n}^{k}$ in (1.1) and the sign $\times$ denotes the Kronecker product. In particular, for $k=1$ we have $n \times n$ matrix derivative from (1.2) whose $(i, j)$-element is $\partial w_{i}(z, \bar{t}) / \partial z_{j}$ $i, j=(1,2, \cdots, n)$, where $\quad w(z, \bar{t})=\left(w_{1}(z, \bar{t}), w_{2}(z, \bar{t}), \cdots, w_{n}(z, \bar{t})\right)^{\prime}$. For a function $w \equiv w(z)$ the $k$-th partial differentiation will be merely denoted as follows: $\partial^{k} w(z) / \partial z^{k}=d^{k} w(z) / d z^{k}$. Moreover, we define the $k$-th power $(d w / d z)^{k}$ of $d w / d z$ as an ${ }_{n} H_{k} \times{ }_{n} H_{k}$ reduced matrix of the $n^{k} \times n^{k}$ matrix $(d w / d z) \times(d w / d z) \times \cdots \times(d w / d z)(\equiv A)$ in the following way:

(i) Construct an $n^{k} \times{ }_{n} H_{k}$ matrix $B$ by adding up the columns in $A$ of which the first elements are equal to each other, and by arranging the above ${ }_{n} H_{k}$ columns so that the first elements of them make a following ${ }_{n} H_{k}$-tuple row vector 


$$
\left(\left(\frac{\partial w_{1}}{\partial z_{1}}\right)^{k}, \cdots, \frac{k !}{k_{1} ! k_{2} ! \cdots k_{n} !}\left(\frac{\partial w_{1}}{\partial z_{1}}\right)^{k_{1}}\left(\frac{\partial w_{1}}{\partial z_{2}}\right)^{k_{2}} \cdots\left(\frac{\partial w_{1}}{\partial z_{n}}\right)^{k_{n}}, \cdots,\left(\frac{\partial w_{1}}{\partial z_{n}}\right)^{k}\right),
$$

where $\left(\partial w_{1} / \partial z_{1}\right)^{k}, \cdots,\left(\partial w_{1} / \partial z_{n}\right)^{k}$ are arranged in the order corresponding to $z_{1}^{k}, \cdots, z_{n}^{k}$ in $(1.1)$.

(ii) Construct ${ }_{n} H_{k} \times{ }_{n} H_{k}$ matrix by leaving only one and removing others among the row vectors in $B$ which are identically equal to each other, and by arranging all remained rows so that the first elements of them make a following ${ }_{n} H_{k}$ column vector

$$
\left(\left(\frac{\partial w_{1}}{\partial z_{1}}\right)^{k}, \cdots,\left(\frac{\partial w_{1}}{\partial z_{1}}\right)^{k_{1}}\left(\frac{\partial w_{2}}{\partial z_{1}}\right)^{k_{2}} \cdots\left(\frac{\partial w_{n}}{\partial z_{1}}\right)^{k_{n}}, \cdots,\left(\frac{\partial w_{n}}{\partial z_{1}}\right)^{k}\right)^{\prime},
$$

where $\left(\partial w_{1} / \partial z_{1}\right)^{k}, \cdots,\left(\partial w_{n} / \partial z_{1}\right)^{k}$ are arranged in the order corresponding to $z_{1}^{k}, \cdots, z_{n}^{k}$ in $(1.1)$ (Ono [11], Tsuboi [13]).

We shall introduce some differential formulas for convenience after calculations. Let the functions $A, B$ of $z$ be $k \times l, l \times m$ matrices, respectively. The following formulas can be easily calculated: $d A B / d z=$ $A(d B / d z)+(d A / d z)\left(E_{n} \times B\right), d A=(d A / d z)\left(d z \times E_{l}\right), d A / d z=(d A / d \zeta)$. $\left((d \zeta / d z) \times E_{l}\right)$, where $E_{n}, E_{l}$ denote $n \times n, l \times l$ unit matrices, respectively.

The following lemmas are trivial.

Lemma 1.1. If for a vector function $w \equiv w(z)$ we have $d w(z) / d z=$ $A$, where $A$ denotes an $n \times n$ constant matrix, then $w(z)=A z+C$, where $C$ is a constant vector of integration.

Lemma 1.2. For a matrix $P \equiv\left(\begin{array}{ll}K & L \\ M & N\end{array}\right)$ with the block subdivisions, where $K, N$ are square matrices, it holds that

$$
P^{-1}=\left(\begin{array}{rr}
K^{-1}+X Z^{-1} Y & -X Z^{-1} \\
-Z^{-1} Y & Z^{-1}
\end{array}\right)
$$

where $X \equiv K^{-1} L, Y \equiv M K^{-1}, Z \equiv N-M K^{-1} L$.

Let $\mathfrak{R}^{2}(D)$ be the class of all functions $w(z)$ which are regular and singlevalued in a domain $D$ and for which the Lebesgue integral $\int_{D}|w(z)|^{2} d v_{z}<\infty$. Then $\mathfrak{2}^{2}(D)$ is a Hilbert space. Since $\mathfrak{2}^{2}(D)$ is separable, there exists a closed orthonormal system $\varphi(z) \equiv\left(\varphi_{1}(z)\right.$, $\left.\varphi_{2}(z), \cdots\right)^{\prime}$. Using this $\varphi(z)$, the Bergman kernel function $k_{D}(z, \bar{t})$ may be represented as follows: $k_{D}(z, \bar{t}) \equiv \varphi^{*}(t) \varphi(z) z, t \in D$. This function is independent of the choice of a closed orthonormal system $p(z)$, and $k_{D}(z, \bar{z})>0$. It is well known that if a function $\zeta \equiv \zeta(z)$ is a pseudo-conformal mapping of a domain $D$ onto a domain $A$, then we have 
$k_{D}(z, \bar{t})=\overline{\operatorname{det} d \tau / d t} \cdot k_{\Delta}(\zeta, \bar{\tau}) \cdot \operatorname{det} d \zeta / d z$, where $\tau \equiv \zeta(t)$. Also, that if we define $T_{D}(z, \bar{z}) \equiv \partial^{2} \log k_{D}(z, \bar{z}) / \partial z^{*} \partial z$, then the $n \times n$ Hermitian matrix $T_{D}(z, \bar{z})$ is transformed under any pseudo-conformal mapping. $\zeta \equiv \zeta(z)$ as a relative invariant; that is, $T_{D}(z, \bar{z})=(d \zeta / d z)^{*} T_{\Delta}(\zeta, \bar{\zeta})(d \zeta / d z)$ and is positive definite. Therefore the Bergman metric

$$
d s^{2} \equiv d z^{*} T_{D}(z, \bar{z}) d z
$$

is absolutely invariant under any pseudo-conformal mapping (Bergman [3]).

Now, we shall define $k_{\mu_{D}}(z, \bar{t}), T_{\mu_{D}}(z, \bar{t})(\mu=1,2)$ as follows, respectively:

$$
\begin{gathered}
T_{\mu_{D}}(z, \bar{t}) \equiv \frac{\partial^{2}}{\partial t^{*} \partial z} \log k_{\mu_{D}}(z, \bar{t}), \quad k_{\varepsilon_{D}}(z, \bar{t}) \equiv \operatorname{det}\left\{k_{1 D}^{2}(z, \bar{t}) T_{1 D}(z, \bar{t})\right\} z, t \in D \\
\left(k_{1 D}(z, \bar{t}) \equiv k_{D}(z, \bar{t})\right),
\end{gathered}
$$

where we assume that $k_{\mu D}(z, \bar{t}) \neq 0$. Then the $n \times n$ matrix functions $T_{D}(z, \bar{t})\left(\equiv T_{1 D}(z, \bar{t})\right)$ and $T_{2 D}(z, \bar{t})$ may be calculated as follows, respectively:

$$
\begin{gathered}
T_{\mu_{D}}(z, \bar{t})=k_{\mu^{-}}^{-2}(z, \bar{t})\left\{k_{\mu_{D}}(z, \bar{t}) \frac{\partial^{2}}{\partial t^{*} \partial z} k_{\mu D}(z, \bar{t})\right. \\
\left.-\frac{\partial}{\partial t^{*}} k_{\mu_{D}}(z, \bar{t}) \frac{\partial}{\partial z} k_{\mu D}(z, \bar{t})\right\} \quad(\mu=1,2) \\
T_{2 D}(z, \bar{t})=2 n \cdot T_{D}(z, \bar{t})+\frac{\partial^{2}}{\partial t^{*} \partial z} \log \operatorname{det} T_{D}(z, \bar{t}) .
\end{gathered}
$$

REMARK 1. As we assume $k_{\mu_{D}}(z, \bar{t}) \neq 0(\mu=1,2)$, our method does not apply to all bounded domains.

LEMma 1.3. $T_{\mu D}(z, \bar{t})(\mu=1,2)$ is relatively invariant under any pseudo-conformal mapping $\zeta \equiv \zeta(z)$, and $T_{2 D}(z, \bar{z})$ is a positive definite Hermitian matrix. Thus we may define the 2-nd invariant metric $d s^{2} \equiv d z^{*} T_{2 D}(z, \bar{z}) d z$.

Proof. $T_{D}(z, \bar{t})$ is relatively invariant (Tsuboi [8]), hence $T_{2 D}(z, \bar{t})$ is also relatively invariant from (1.4). Thus, it holds that

$$
T_{\mu D}(z, \bar{t})=\left(\frac{d \tau}{d t}\right)^{*} T_{\mu \Delta}(\zeta, \bar{\tau}) \frac{d \zeta}{d z} \quad(\mu=1,2),
$$

where $\tau \equiv \zeta(t)$ and $\Delta \equiv \zeta(D)$. Next it has been known that the matrix

$$
(n+1)\left(g_{\bar{\alpha} \beta}\right)-\left(R_{\bar{\alpha} \beta}\right)(\alpha, \beta=1,2, \cdots, n)
$$


is positive definite, where $g_{\bar{\alpha} \beta}$ and $R_{\bar{\alpha} \beta}$ are covariant metric tensor and the Ricci curvature tensor for bounded complex manifold, respectively (Kobayashi [5]). In our case, we can take $T_{D}(z, \bar{z})$ as $\left(g_{\bar{\alpha} \beta}\right)$ and $-\partial^{2} \log \operatorname{det} T_{D}(z, \bar{z}) / \partial z^{*} \partial z$ as $\left(R_{\bar{\alpha} \beta}\right)$. Hence (1.6) becomes as follows:

$$
u^{*}\left[(n+1) T_{D}(z, \bar{z})+\frac{\partial^{2}}{\partial z^{*} \partial z} \log \operatorname{det} T_{D}(z, \bar{z})\right] u>0,
$$

where $u$ is an arbitrary $n$-tuple nonzero constant vector. We utilize this relation (1.7). By (1.4) we have

$$
\begin{aligned}
u^{*} T_{2 D}(z, \bar{z}) u & =u^{*}\left[2 n \cdot T_{D}(z, \bar{z})+\frac{\partial^{2}}{\partial z^{*} \partial z} \log \operatorname{det} T_{D}(z, \bar{z})\right] u \\
& \geqq u^{*}\left[(n+1) T_{D}(z, \bar{z})+\frac{\partial^{2}}{\partial z^{*} \partial z} \log \operatorname{det} T_{D}(z, \bar{z})\right] u>0,
\end{aligned}
$$

the proof is completed.

If $D$ and $\Delta$ are domains which are pseudo-conformally equivalent to each other by $\zeta \equiv \zeta(z)$, then for the scalar function $k_{2 D}(z, \bar{t})$ we have

$$
k_{2 D}(z, \bar{t})=\left(\overline{\operatorname{det} \frac{d \tau}{d t}}\right)^{m} k_{2 \Delta}(\zeta, \bar{\tau})\left(\operatorname{det} \frac{d \zeta}{d z}\right)^{m}, \quad m=1+2 n
$$

and it is evident that $k_{2 D}(z, \bar{z})$ is positive from its definition.

2. $\mu$-th Representative domains and $\mu$-th quasiminimal domains $(\mu=1,2)$. Generalization of the Riemann mapping theorem to the case of domains in the spase of several complex variables leads to various other types of canonical domains.

Firstly we shall introduce the generalized representative domain. We define the vector function $M_{\mu D}^{O E n}\left(z, t_{0}\right)$ and the scalar function $m_{\mu D}^{1}\left(z, t_{0}\right)$ in $D$ for a fixed point $t_{0}(\in D)$, which is not a point on a branch manifold, as

$$
M_{\mu D}^{o E_{n}}\left(z, t_{0}\right) \equiv\left(0 E_{n}\right)\left(\begin{array}{cc}
k_{\mu_{D}}\left(t_{0}, \bar{t}_{0}\right) & \frac{\partial}{\partial z} k_{\mu_{D}}\left(t_{0}, \bar{t}_{0}\right) \\
\frac{\partial}{\partial t^{*}} k_{\mu_{D}}\left(t_{0}, \bar{t}_{0}\right) & \frac{\partial^{2}}{\partial t^{*} \partial z} k_{\mu_{D}}\left(t_{0}, \bar{t}_{0}\right)
\end{array}\right)^{-1}\left(\begin{array}{l}
k_{\mu_{D}}\left(z, \bar{t}_{0}\right) \\
\frac{\partial}{\partial t^{*}} k_{\mu_{D}}\left(z, \bar{t}_{0}\right)
\end{array}\right)
$$

and $m_{\mu_{D}}^{1}\left(z, t_{0}\right) \equiv k_{\mu_{D}}\left(z, \bar{t}_{0}\right) / k_{\mu_{D}}\left(t_{0}, \bar{t}_{0}\right)$, respectively, where 0 denotes an $n$ tuple column zero-vector. Hereafter we assume that $\operatorname{det} T_{\mu D}\left(z, \bar{t}_{0}\right) \neq 0$ for all $z \in D$.

REMARK 2. Let $P^{-1}$ be the second matrix of right side in (2.1), then by a simple calculation of determinants we have 


$$
\operatorname{det} P=k_{\mu D}^{n+1}\left(t_{0}, \bar{t}_{0}\right) \operatorname{det} T_{\mu D}\left(t_{0}, \bar{t}_{0}\right)>0 .
$$

Hence there exists the inverse of $P$. Further from the rules of matrices we see that $M_{\mu D}^{o E} n\left(t_{0}, t_{0}\right)=0$.

Lemma 2.1. The matrix derivative $d\left(M_{\mu D}^{o E_{n}} / m_{\mu D}^{1}\right) / d z$ is given by the matrix product

$$
\frac{d}{d z} \frac{M_{\mu D}^{o E_{n}}\left(z, t_{0}\right)}{m_{\mu D}^{1}\left(z, t_{0}\right)}=T_{\mu D}^{-1}\left(t_{0}, \bar{t}_{0}\right) T_{\mu D}\left(z, \bar{t}_{0}\right)
$$

Proof. Using Lemma 1.2 and the relation (1.3), further noting the definition of $T_{\mu D}(z, \bar{t})$ the left side of (2.2) is calculated as follows:

$$
k_{\mu_{D}}\left(t_{0}, \bar{t}_{0}\right)\left(0 E_{n}\right)\left({ }^{*} T_{\mu D}^{-1}\left(t_{0}, \bar{t}_{0}\right) / k_{\mu D}^{*}\left(t_{0}, \bar{t}_{0}\right)\right)\left(0 T_{\mu D}^{\prime}\left(z, \bar{t}_{0}\right)\right)^{\prime} .
$$

From this we obtain the desired relation $T_{\mu D}^{-1}\left(t_{0}, \bar{t}_{0}\right) T_{\mu D}\left(z, \bar{t}_{0}\right)$.

Theorem 2.1. If a domain $D$ is mapped onto a domain $\triangle$ by a pseudo-conformal mapping $\zeta=\zeta(z)$ which satisfies $\zeta\left(t_{0}\right)=\tau_{0}, d \zeta\left(t_{0}\right) / d z=$ $E_{n}$ at a fixed point $t_{0}(\in D)$, then we have

$$
\frac{M_{\mu \nu}^{0 E n}\left(z, t_{0}\right)}{m_{\mu D}^{1}\left(z, t_{0}\right)}=\frac{M_{\mu \Delta}^{o E_{n}}\left(\zeta(z), \tau_{0}\right)}{m_{\mu \Delta}^{1}\left(\zeta(z), \tau_{0}\right)} .
$$

Thus by the function $\left(M_{\mu}^{0 E_{n}} / m_{\mu}^{1}\right)+v_{0} D$ and $\triangle$ generate the same domain $B\left(\ni v_{0}(\right.$ not located on a branch manifold)) of certain kind.

Proof. Integrating from $t_{0}$ to $z$ both sides of (2.2), and noting $M_{\mu D}^{o E} n\left(t_{0}, t_{0}\right) / m_{\mu D}^{1}\left(t_{0}, t_{0}\right)=0$ the vector function $M_{\mu_{D}} / m_{\mu_{D}}$ is represented by

$$
\frac{M_{\mu D}^{0 E} n\left(z, t_{0}\right)}{m_{\mu D}^{1}\left(z, t_{0}\right)}=T_{\mu D}^{-1}\left(t_{0}, \bar{t}_{0}\right) \int_{t_{0}}^{z} T_{\mu D}\left(z, \bar{t}_{0}\right) d z
$$

Using the relations (2.4) and (1.5), the invariant (2.3) is easily obtained.

We call this unique domain $B$ (in Theorem 2.1) a $\mu$-th representative domain of $D$ with respect to $t_{0}$ with center at a point $v_{0}$. Further we shall name $w(z)=\left(M_{\mu D}^{0 E_{n}}\left(z, t_{0}\right) / m_{\mu D}^{1}\left(z, t_{0}\right)\right)+v_{0}$ a $\mu$-th representative function of the domain $D$.

If a domain $D$ is homogeneous, then we have $\operatorname{det} T_{D}(z, \bar{t})=$ $c \cdot k_{D}(z, \bar{t})$ in $D$. Hence $T_{2 D}(z, \bar{t})$ is equal to $(2 n+1) T_{D}(z, \bar{t})$. Thus in this case 2 -nd representative function coincides with the usual representative function.

We shall consider the mapping of $D$ onto $B$ by means of the function $w=\left(M_{\mu D}^{0 E} n\left(z, t_{0}\right) / m_{\mu D}^{1}\left(z, t_{0}\right)\right)+v_{0}$. Then the domain $B$ becomes 
a $\mu$-th representative domain with center at $v_{0}$, and further it holds that $\left(M_{\mu D}^{0 E} n\left(z, t_{0}\right) / m_{\mu D}^{1}\left(z, t_{0}\right)\right)+v_{0}=\left(M_{\mu B}^{0 E n}\left(w, v_{0}\right) / m_{\mu B}^{1}\left(w, v_{0}\right)\right)+v_{0}$ by Theorem 2.1 because $w(z)$ satisfies the normalized conditions $w\left(t_{0}\right)=v_{0}, d w\left(t_{0}\right) / d z=$ $E_{n}$. Therefore we have

CoRollary 2.1. $\mu$-th Representative function of a $\mu$-th representative domain $B$ is the identity function.

Corollary 2.2. A domain $B$ is a $\mu$-th representative domain with center at $v_{0}$ if and only if

$$
T_{\mu_{B}}\left(w, \bar{v}_{0}\right)=T_{\mu_{B}}\left(v_{0}, \bar{v}_{0}\right)=\text { const. for } w \in B .
$$

Proof. Suppose that $B$ is a $\mu$-th representative domain with center at $v_{0}$. Then from the property of $\mu$-th representative domain, it holds that $w-v_{0}=M_{\mu_{B}}^{0 E} n\left(w, v_{0}\right) / m_{\mu B}^{1}\left(w, v_{0}\right)$. By differentiating this equation with respect to $w$, we obtain $E_{n}=T_{\mu B}^{-1}\left(v_{0}, \bar{v}_{0}\right) T_{\mu B}\left(w, \bar{v}_{0}\right)$ from (2.2). Suppose conversely that the relation (2.5) holds. Let $B_{1}$ be the $\mu$-th representative domain of $B$ with respect to $v_{0}$, that is, the image domain by the $\mu$-th representative function $w_{1}(w)$ of $B$. Then it suffices to show that $w_{1}(w)$ is equal to $w$. Since

$$
d w_{1}(w) / d w=T_{\mu B}^{-1}\left(v_{0}, \bar{v}_{0}\right) T_{\mu B}\left(w, \bar{v}_{0}\right)
$$

by Lemma 2.1, we have $d w_{1}(w) / d w=E_{n}$ from our assumption (2.5). Therefore, $w_{1}(w)=w$ because $w_{1}\left(v_{0}\right)=v_{0}$ (See Lemma 1.1).

A domain $B$ is minimal with center $v_{0}$ if and only if $k_{B}\left(w, \bar{v}_{0}\right)$ is constant for $w \in B$. In view of this fact we shall define the $\mu$-th quasiminimal domain which is the generalization of minimal domain, and secondly we shall state some results.

A domain $B$ is called a $\mu$-th quasiminimal domain with center at a point $v_{0}(\in B)$ if and only if $k_{\mu_{B}}\left(w, \bar{v}_{0}\right)=k_{\mu_{B}}\left(v_{0}, \bar{v}_{0}\right)=$ const. for $w \in B$. Therefore the 1-st quasiminimal domain coincides with the ordinary minimal domain.

Let a domain $B$ be minimal and representative with the same center at $v_{0}$, then $k_{B}\left(w, \bar{v}_{0}\right)=$ const. and $T_{B}\left(w, \bar{v}_{0}\right)=$ const. for $w \in B$, respectively (Maschler [12], Tsuboi [8]). Hence we have $k_{2 B}\left(w, \bar{v}_{0}\right)=$ const. Thus there exists the 2-nd quasiminimal domain.

THEOREM 2.2. A necessary and sufficient condition that a $\mu$-th representative domain $B$ of a domain $D$ with respect to $t_{0}$ with center at $v_{0}$ becomes simultaneously a $\mu-t h$ quasiminimal domain with the same center $v_{0}$ is 
$\left(\operatorname{det} T_{\mu_{D}}\left(z, \bar{t}_{0}\right)\right)^{m} / k_{\mu_{D}}\left(z, \bar{t}_{0}\right)=\left(\operatorname{det} T_{\mu D}\left(t_{0}, \bar{t}_{0}\right)\right)^{m} / k_{\mu_{D}}\left(t_{0}, \bar{t}_{0}\right)=$ const ., where we take $m=1,1+2 n$ for $\mu=1,2$, respectively.

Proof. According to the invariant relations (1.5) and (1.8), we have $\left(\operatorname{det} T_{\mu_{D}}\left(z, \bar{t}_{0}\right)\right)^{m} / r_{\mu_{D}}\left(z, \bar{t}_{0}\right)=\left(\operatorname{det} T_{\mu_{B}}\left(w, \bar{v}_{0}\right)\right)^{m} / k_{\mu_{B}}\left(w, \bar{v}_{0}\right)$,

$$
\left(\operatorname{det} T_{\mu_{D}}\left(t_{0}, \bar{t}_{0}\right)\right)^{m} / k_{\mu_{D}}\left(t_{0}, \bar{t}_{0}\right)=\left(\operatorname{det} T_{\mu_{B}}\left(v_{0}, \bar{v}_{0}\right)\right)^{m} / k_{\mu_{B}}\left(v_{0}, \bar{v}_{0}\right)
$$

for a $\mu$-th representative function $w=w(z)$ of $D$. From this formulas our required result is directly obtained.

THEOREM 2.3. A necessary and sufficient condition that a $\mu$-th quasiminimal domain $B$ with center at $v_{0}$ becomes simultaneously a $\mu$-th representative domain with the same center $v_{0}$ is

$$
\partial^{2} k_{\mu_{B}}\left(w, \bar{v}_{0}\right) / \partial v^{*} \partial w=k_{\mu_{B}}\left(v_{0}, \bar{v}_{0}\right) T_{\mu_{B}}\left(v_{0}, \bar{v}_{0}\right)=\text { const } .
$$

Proof. Since $B$ is a $\mu$-th quasiminimal domain, we have

$$
T_{\mu_{B}}\left(w, \bar{v}_{0}\right)=k_{\mu_{B}}^{-1}\left(v_{0}, \bar{v}_{0}\right) \frac{\partial^{2}}{\partial v^{*} \partial w} k_{\mu_{B}}\left(w, \bar{v}_{0}\right)
$$

from (1.3) and the equation $\partial k_{\mu_{B}}\left(w, \bar{v}_{0}\right) / \partial w=0$ which is equivalent to $k_{\mu_{B}}\left(w, \bar{v}_{0}\right)=$ const. From (2.6) and Corollary 2.2, our desired conclusion is at once obtained.

For $\mu=1$ we have the following corollary.

COROLLARY 2.3. A necessary and sufficient condition that a minimal domain $B$ with center at $v_{0}$ becomes simultaneously $a$ representative domain with the same center $v_{0}$ is

$$
\frac{\partial^{2}}{\partial v^{*} \partial w} k_{B}\left(w, \bar{v}_{0}\right)=k_{B}\left(v_{0}, \bar{v}_{0}\right) T_{B}\left(v_{0}, \bar{v}_{0}\right)=\text { const } .
$$

If we denote $A \equiv k_{B}\left(v_{0}, \bar{v}_{0}\right) T_{B}\left(v_{0}, \bar{v}_{0}\right)$ in Corollary 2.3 , then $A$ is a positive definite constant Hermitian matrix. Using this $A$ the condition (2.7) may be described as $\partial^{2} k_{B}\left(w, \bar{v}_{0}\right) / \partial v^{*} \partial w=A$. This is equivalent to $\partial k_{B}\left(w, \bar{v}_{0}\right) / \partial v^{*}=A\left(w-v_{0}\right)$ because $\partial k_{B}\left(v_{0}, \bar{v}_{0}\right) / \partial v^{*}=0$ (See Maschler [12]).

ExAmple 1. Let $D$ be a bounded domain in $z$-plane. Then there exists a unique function $w \equiv w(z)$ which maps $D$ onto the 2-nd quasiminimal domain $B$ with center at $v_{0}\left(=w\left(t_{0}\right)\right)$ and it is 


$$
w(z)=k_{2 D}^{-1 / 3}\left(t_{0}, \bar{t}_{0}\right) \int_{t_{0}}^{z} k_{2 D}^{1 / 3}\left(z, \bar{t}_{0}\right) d z+v_{0} .
$$

In fact, using the invariant $(1.8)(n=1)$ we have

$$
k_{2 D}^{-1 / 3}\left(t_{0}, \bar{t}_{0}\right) \int_{t_{0}}^{z} k_{2 D}^{1 / 3}\left(z, \bar{t}_{0}\right) d z+v_{0}=k_{2 B}^{-1 / 3}\left(v_{0}, \bar{v}_{0}\right) \int_{v_{0}}^{w} k_{2 B}^{1 / 3}\left(w, \bar{v}_{0}\right) d w+v_{0} .
$$

From this relation, (2.8) is easily obtained.

REMARK 3. We see from (2.9) that the function (2.8) is also a kind of representative function of the domain $D$. In general, the function $w(z) \equiv \int_{t_{0}}^{z}\left\{m_{\mu D}^{10 \cdots 0}\left(z, t_{0}\right)\right\}^{1 / \nu} d z(\nu=1,3$ for $\mu=1,2)$ in the case of one variable may become a $\mu$-th $m$-representative function (See $\S 4$ ).

3. $\mu$-th Normal domains $(\mu=1,2)$. We denote $N_{\mu D}^{E_{n}}\left(z, t_{0}\right) \equiv$ $T_{\mu D}^{-1}\left(t_{0}, \bar{t}_{0}\right) T_{\mu D}\left(z, \bar{t}_{0}\right), \widetilde{M}_{\mu D}^{0 E_{n}}\left(z, t_{0}\right) \equiv \int_{t_{0}}^{z} N_{\mu D}^{E_{n}}\left(z, t_{0}\right) d z$ (See $\left.\S 4\right)$, and we shall consider the function

$$
w(z)=T_{\mu D}^{1 / 2}\left(t_{0}, \bar{t}_{0}\right) \tilde{M}_{\mu D}^{0 E_{n}}\left(z, t_{0}\right)+v_{0} .
$$

Then from (1.5) we have

$$
\begin{aligned}
d w^{*} d w & =d z^{*} N_{\mu D}^{E_{n}}\left(z, t_{0}\right)^{*} T_{\mu D}\left(t_{0}, \bar{t}_{0}\right) N_{\mu D}^{E_{n}}\left(z, t_{0}\right) d z \\
& =d \zeta * N_{\mu \Delta}^{E_{n}}\left(\zeta(z), \tau_{0}\right)^{*} T_{\mu \Delta}\left(\tau_{0}, \bar{\tau}_{0}\right) N_{\mu \Delta}^{E_{n}}\left(\zeta(z), \tau_{0}\right) d \zeta
\end{aligned}
$$

for any pseudo-conformal mapping $\zeta \equiv \zeta(z)$. Hence we obtain

$$
(d w \equiv) T_{\mu D}^{1 / 2}\left(t_{0}, \bar{t}_{0}\right) N_{\mu D}^{E_{n}}\left(z, t_{0}\right) d z=U \cdot T_{\mu\lrcorner}^{1 / 2}\left(\tau_{0}, \bar{\tau}_{0}\right) N_{\mu \Delta}^{E_{n}}\left(\zeta(z), \tau_{0}\right) d \zeta,
$$

where $U$ is a constant unitary matrix depending upon the points $t_{0}$ and $\tau_{0}$. Thus we have

THEOREM 3.1. The function (3.1) and the domain $B \equiv w(D)$ are both invariant under any pseudo-conformal mapping $\zeta \equiv \zeta(z)$, up to the constant unitary matrices.

The above uniquely determined domain $B$ up to the constant unitary matrix is called a $\mu$-th normal domain of $D$ with center at a point $v_{0}$.

CoRollary 3.1. A domain $B$ is a $\mu$-th normal domain with center at $v_{0}$ if and only if

$$
N_{\mu_{B}}^{E_{n}}\left(w, v_{0}\right)=T_{\mu_{B}}^{-1 / 2}\left(v_{0}, \bar{v}_{0}\right) \cdot U^{*}=\text { const. for } w \in B \text {. }
$$

Proof. Let the domain $B$ be a $\mu$-th normal domain of a domain 
$D$. Then the function $w(z)=T_{\mu D}^{1 / 2}\left(t_{0}, \bar{t}_{0}\right) \widetilde{M}_{\mu_{D}}^{0 E} n\left(z, t_{0}\right)+v_{0}$ maps $D$ onto $B$. For this $w(z)$ it holds from (3.2) that

$$
T_{\mu D}^{1 / 2}\left(t_{0}, \bar{t}_{0}\right) \tilde{M}_{\mu D}^{0 E n}\left(z, t_{0}\right)+v_{0}=U \cdot T_{\mu B}^{1 / 2}\left(v_{0}, \bar{v}_{0}\right) \tilde{M}_{\mu B}^{0 E n}\left(w, v_{0}\right)+v_{0} .
$$

Therefore we have $w=U \cdot T_{\mu_{B}}^{1 / 2}\left(v_{0}, \bar{v}_{0}\right) \widetilde{M}_{\mu_{B}}^{0 E_{n}}\left(w, v_{0}\right)+v_{0}$. Differentiating this equation concerning $w$, we obtain $E_{n}=U \cdot T_{\mu_{B}}^{1 / 2}\left(v_{c}, \bar{v}_{0}\right) N_{\mu B}^{E_{n}}\left(w, v_{0}\right)$. Conversely if (3.3) holds, then

$$
U \cdot T_{\mu B}^{1 / 2}\left(v_{0}, \bar{v}_{0}\right) \widetilde{M}_{\mu B}^{0 E} n\left(w, v_{0}\right)=\int_{v_{0}}^{w} E_{n} d w=w-v_{0} .
$$

Corollary 3.2. If a domain $B$ is a $\mu$-th normal domain with center at $v_{0}$, then we have $T_{\mu B}\left(w, \bar{v}_{0}\right)=E_{n}$.

The proof is obvious.

4. $\mu$-th $m$-Representative domains $(\mu=1,2)$. We shall investigate the generalized $m$-representative domains using the invariant of certain kind which is constructed by $T_{\mu D}(z, \bar{t})$ and its derivatives.

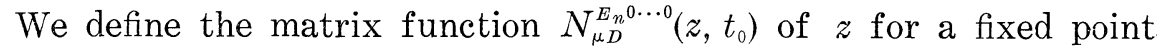
$t_{0}(\in D)$ as follows:

$$
N_{\mu D}^{E_{n} 0 \cdots 0}\left(z, t_{0}\right)
$$

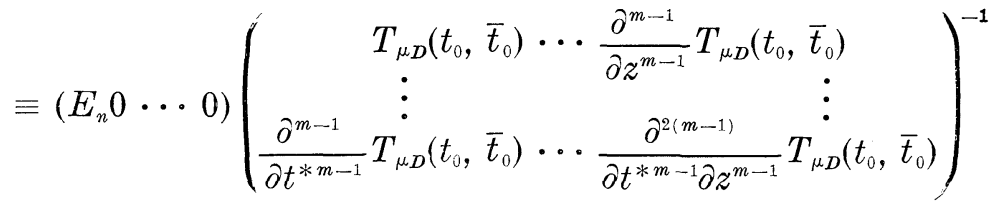

$$
\begin{aligned}
& \cdot\left(\begin{array}{c}
T_{\mu D}\left(z, \bar{t}_{0}\right) \\
\vdots \\
\frac{\partial^{m-1}}{\partial t^{* m-1}} T_{\mu D}\left(z, \bar{t}_{0}\right)
\end{array}\right)
\end{aligned}
$$

where several zeros denote $n \times{ }_{n} H_{2}, \cdots, n \times{ }_{n} H_{m}$ zero-matrices, respectively. And we denote the vector function

$$
\widetilde{M}_{\mu D}^{0 E_{n}{ }^{0 \cdots 0}}\left(z, t_{0}\right) \equiv \int_{t_{0}}^{z} N_{\mu D}^{E_{n}{ }^{0 \cdots 0}}\left(z, t_{0}\right) d z
$$

Here we assume that there exists the inverse of the second matrix

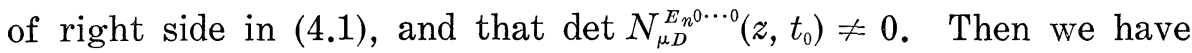

THEOREM 4.1. The function $w(z)=\widetilde{M}_{\mu D}^{0 n_{n}{ }^{0 \cdots 0}}\left(z, t_{0}\right)$ satisfies the normalized conditions

$$
w\left(t_{0}\right)=0, \quad \frac{d w\left(t_{0}\right)}{d z}=E_{n}, \quad \frac{d^{2} w\left(t_{0}\right)}{d z^{2}}=\cdots=\frac{d^{m} w\left(t_{0}\right)}{d z^{m}}=0 .
$$


And if a domain $D$ is mapped onto a domain $\triangle$ by a pseudo-conformal mapping $\zeta \equiv \zeta(z)$ which satisfies

$$
\zeta\left(t_{0}\right)=\tau_{0}, \quad d \zeta\left(t_{0}\right) / d z=E_{n}, \quad d^{2} \zeta\left(t_{0}\right) / d z^{2}=\cdots=d^{m} \zeta\left(t_{0}\right) / d z^{m}=0
$$

at a fixed point $t_{0}$, then we have

$$
\widetilde{M}_{\mu D}^{0 E n^{0 \cdots 0}}\left(z, t_{0}\right)=\widetilde{M}_{\mu \perp}^{0 E n^{0 \cdots 0}}\left(\zeta(z), \tau_{0}\right) .
$$

Thus by the function $\widetilde{M}_{\mu}^{0 E_{n}{ }^{0 \cdots 0}}+v_{0} D$ and $\Delta$ generate the same domain $B$.

Proof. The relation (4.2) is easily obtained from (4.1) by $(N$, $\left.d N / d z, d^{2} N / d z^{2}, \cdots, d^{m-1} N / d z^{m-1}\right)=\left(E_{n}, 0, \cdots, 0\right)$, where

$$
N \equiv N_{\mu D}^{E_{n} 0 \cdots 0}\left(t_{0}, t_{0}\right) \text {. }
$$

Next, noting $\partial T / \partial z=\partial^{3} \log k / \partial t^{*} \partial z^{2}, \cdots$, etc., we have the following relations for a pseudo-conformal mapping $\zeta \equiv \zeta(z)$ satisfying

$$
\zeta\left(t_{0}\right) \equiv \tau_{0}, \quad d^{2} \zeta\left(t_{0}\right) / d z^{\mathrm{N}}=\cdots=d^{m \zeta}\left(t_{0}\right) / d z^{m}=0
$$

at $t_{0}$ :

$$
\partial^{p+q} T_{\mu D}\left(z, \bar{t}_{0}\right) / \partial t^{* p} \partial z^{q}=\left(d \tau\left(t_{0}\right) / d t\right)^{* p+1} \cdot \hat{o}^{p+q} T_{\mu\lrcorner}\left(\zeta, \bar{\tau}_{0}\right) / \partial \tau^{* p} \widehat{\sigma}^{\zeta q} \cdot(d \zeta(z) / d z)^{q+1},
$$

where $p, q(0 \leqq p, q \leqq m-1)$ are both integers. Hence we obtain

$$
N_{\mu D}^{E_{n} 0 \cdots 0}\left(z, t_{0}\right)=\left(\frac{d \zeta\left(t_{0}\right)}{d z}\right)^{-1} N_{\mu d}^{E_{n} 0 \cdots 0}\left(\zeta(z), \tau_{0}\right) \frac{d \zeta(z)}{d z},
$$

which is led by a simple calculation (This relation (4.4) is important for study of $\mu$-th $m$-normal domains). Integrating (4.4) from $t_{0}$ to $z$ and noting our added assumption $d \zeta\left(t_{0}\right) / d z=E_{n}$, we have the required result (4.3).

We call, similarly in $\S 2$, the unique domain $B$ (in Theorem 4.1) a $\mu$-th $m$-representative domain $(m \geqq 1)$ of $D$ with respect to $t_{0}$ with center at a point $v_{0}$. Further we shall name $w(z)=\widetilde{M}_{\mu D}^{0 E n^{0 \cdots 0}}\left(z, t_{0}\right)+v_{0}$ a $\mu$-th m-representative function of the domain $D$.

EXAMPle 2. For a unit circle in the case of one variable, the 1-st 2-representative function with respect to $t_{0}\left(v_{0}=0\right)$ is

$$
w(z)=\left(1-\left|t_{0}\right|^{2}\right)\left(1-\bar{t}_{0} u\right) u,
$$

where $u \equiv\left(z-t_{0}\right) /\left(1-\bar{t}_{0} z\right)$. The other side Maschler's (1-st) 2-representative function of a unit circle is calculated as follows:

$$
w(z)=\left(1-\left|t_{0}\right|^{2}\right)\left(1-3 \bar{t}_{0} u\right) u /\left(1-2 \bar{t}_{0} u\right) .
$$


In this case if we choose a fixed point $t_{0}$ in $1 / 2<\left|t_{0}\right|<1$, then $w(z)$ is nonregular.

COROLLARY 4.1. $\mu$-th m-Representative function of a $\mu$-th $m$ representative domain $B$ is the indentity function.

This corollary is proved similarly as the proof of Corollary 2.1. Furthermore, as well as Corollary 2.2, we have

CoRollary 4.2. A domain $B$ is a $\mu$-th m-representative domain with center at $v_{0}$ if and only if $N_{\mu_{B}}^{E_{n} 0 \cdots 0}\left(w, v_{0}\right)=E_{n}\left(=N_{\mu B}^{E_{n} 0 \cdots 0}\left(v_{0}, v_{0}\right)\right)$ for $w \in B$.

Let $B$ be a $\mu$-th $m$-representative domain with center at $v_{0}$, then, in the relation

$$
N_{\mu_{B}}^{E_{n} 0 \cdots 0}\left(w, v_{0}\right)=N_{\mu_{B}}^{E_{n} 0 \cdots 0}\left(w, v_{0}\right)+\left(\widehat{o}^{m} N_{\mu B}^{E_{n} 0 \cdots 0}\left(v_{0}, v_{0}\right) / \partial w^{m}\right)(*)
$$

which is obtained by a simple calculation using Lemma 1.2, we have $N_{\mu B}^{E_{n} 0 \cdots 0}\left(w, v_{0}\right)=E_{n}$ from Corollary 4.2 so that

$$
\partial^{m} N_{\mu B}^{E_{n}{ }^{0 \cdots 0}}\left(w, v_{0}\right) / \partial w^{m}
$$

vanishes at $v_{0}$. Thus we conclude $N_{\mu B}^{E_{n}{ }^{0 \cdots 0}}\left(w, v_{0}\right)=E_{n}$. Summing up the above argument we have arrived at

THEOREM 4.2. If a domain $B$ is a $\mu$-th m-representative domain with center at $v_{0}$, then $B$ is also a $\mu$-th $(m+1)$-representative domain with the same center $v_{0}$.

The author wishes to express here his hearty gratitude to Prof. S. Ozaki for his kind guidance during his research.

\section{REFERENCES}

1. S. Bergman, Sur les fonctions orthogonales de plusieurs variables complexes avec les 1 applications à la théorie des fonctions analytiques, Mém. Sci. Math. 106 (1947), 40-57. 2.—_ Sur la fonction-noyau d'un domaine et ses applications dans la théorie des transformations pseudo-conformes, Mém. Sci. Math. 108 (1948), 27-42.

3. - The kernel function and conformal mapping, Amer. Math. Soc., New York, 1950.

4. - Über die Kernfunktion gewisser Reinhardtschen Kreiskörper, Rev. Math. de 1'Union Interbalcanique 2 (1939), 41-43.

5. S. Kobayashi, Geometry of bounded domains, Trans. Amer. Math. Soc. 92 (1959), 267-290.

6. S. Katō, Theorems in several complex variables, Rep. Fac. Tech. Kanagawa Univ.

4 (1965), 7-27.

7. M. Maschler, Classes of minimal and representative domains and their kernel func- 
tions, Pacific J. Math. 9 (1959), 763-781.

8. T. Tsuboi, Bergman representative domains and minimal domains, Jap. J. Math. 29 (1959), 141-148.

9. S. Kakurai, Characterization of canonical domains of Bergman and analytic automorphisms with an invariant point, Sci. Rep. Tokyo Kyoiku D. A. 7 (1963), 275-307. 10. M. Sugawara, On the theory of kernel functions, J. Fac. Sci. Univ. Tokyo, Sect. I, 7 (1956), 265-303.

11. I. Ono, Analytic vector functions of several complex variables, J. Math. Soc. Japan, 8 (1956), 216-246.

12. M. Maschler, Minimal domains and their Bergman kernel function, Pacific J. Math. 6 (1956), 501-516.

13. T. Tsuboi, $n$-Representative domains and n-minimal domains in several complex variables, Sci. Rep. Saitama Univ. A. 4 (1961), 1-14.

14. S. Ozaki, I. Ono and T. Umezawa, General minimum problems and representative domains, Sci. Rep. Tokyo Kyoiku Daigaku A. 5 (1955), 1-7.

15. Y. Michiwaki, On the theory of analytic functions of several complex variables, Sci. Rep. Tokyo Kyoiku D. A. 6 (1959), 263-279.

Received November 11, 1964, and in revised form February 9, 1966.

Kanagawa University, Yokohama, JaPan 



\section{PACIFIC JOURNAL OF MATHEMATICS}

\section{EDITORS}

H. SAMELSON

Stanford University

Stanford, California

J. P. JANS

University of Washington

Seattle, Washington 98105

\section{J. DugunduI}

University of Southern California Los Angeles, California 90007

RICHARD ARENS

University of California

Los Angeles, California 90024

\section{ASSOCIATE EDITORS}
E. F. BECKENBACH
B. H. NEUMANN
F. WOLF
K. YoSIDA

\section{SUPPORTING INSTITUTIONS}

\author{
UNIVERSITY OF BRITISH COLUMRIA \\ CALIFORNIA INSTITUTE OF TECHNOLOGY \\ UNIVERSITY OF CALIFORNIA \\ MONTANA STATE UNIVERSITY \\ UNIVERSITY OF NEVADA \\ NEW MEXICO STATE UNIVERSITY \\ OREGON STATE UNIVERSITY \\ UNIVERSITY OF OREGON \\ OSAKA UNIVERSITY \\ UNIVERSITY OF SOUTHERN CALIFORNIA
}

\author{
STANFORD UNIVERSITY \\ UNIVERSITY OF TOKYO \\ UNIVERSITY OF UTAH \\ WASHINGTON STATE UNIVERSITY \\ UNIVERSITY OF WASHINGTON \\ AMERICAN MATHEMATICAL SOCIETY \\ CHEVRON RESEARCH CORPORATION \\ TRW SYSTEMS \\ NAVAL ORDNANCE TEST STATION
}

Mathematical papers intended for publication in the Pacific Journal of Mathematics should be typewritten (double spaced). The first paragraph or two must be capable of being used separately as a synopsis of the entire paper. It should not contain references to the bibliography. Manuscripts may be sent to any one of the four editors. All other communications to the editors should be addressed to the managing editor, Richard Arens at the University of California, Los Angeles, California 90024 .

50 reprints per author of each article are furnished free of charge; additional copies may be obtained at cost in multiples of 50 .

The Pacific Journal of Mathematics is published monthly. Effective with Volume 16 the price per volume (3 numbers) is $\$ 8.00$; single issues, $\$ 3.00$. Special price for current issues to individual faculty members of supporting institutions and to individual members of the American Mathematical Society: $\$ 4.00$ per volume; single issues $\$ 1.50$. Back numbers are available.

Subscriptions, orders for back numbers, and changes of address should be sent to Pacific Journal of Mathematics, 103 Highland Boulevard, Berkeley 8, California.

Printed at Kokusai Bunken Insatsusha (International Academic Printing Co., Ltd.), No. 6, 2-chome, Fujimi-cho, Chiyoda-ku, Tokyo, Japan.

\section{PUBLISHED BY PACIFIC JOURNAL OF MATHEMATICS, A NON-PROFIT CORPORATION}

The Supporting Institutions listed above contribute to the cost of publication of this Journal, but they are not owners or publishers and have no responsibility for its content or policies. 


\section{Pacific Journal of Mathematics}

\section{Vol. 21, No. 2 December, 1967}

Arne P. Baartz, The measure algebra of a locally compact semigroup ..... 199

Robert F. Brown, On maps with identical fixed point sets............. 215

C. Buttin, Existence of a homotopy operator for Spencer's sequence in the analytic case ..................................... 219

Henry Werner Davis, An elementary proof that Haar measurable almost periodic functions are continuous ........................ 241

Zeev Ditzian, On asymptotic estimates for kernels of convolution transforms ...................................... 249

Robert E. Edwards, Boundedness principles and Fourier theory ......... 255

John A. Hildebrant, On compact unithetic semigroups ............... 265

Marinus A. Kaashoek and David Clark Lay, On operators whose Fredholm set is the complex plane ............................ 275

Sadao Kató, Canonical domains in several complex variables ........... 279

David Clifford Kay, The ptolemaic inequality in Hilbert geometries.... . . . 293

Joseph D. E. Konhauser, Biorthogonal polynomials suggested by the Laguerre polynomials ............................. 303

Kevin Mor McCrimmon, Macdonald's theorem with inverses .......... 315

Harry Eldon Pickett, Homomorphisms and subalgebras of multialgebras .................................... 327

Richard Dennis Sinkhorn and Paul Joseph Knopp, Concerning nonnegative matrices and doubly stochastic matrices ..............

Erling Stormer, On anti-automorphisms of von Neumann algebras ...

Miyuki Yamada, Regular semi-groups whose idempotents satisfy permutation identities .......................... 